\title{
State of the art lecture: Lithiasis and pancreatitis
}

\author{
B. Napoléon ${ }^{1,2}$, C. Lefort ${ }^{1,2}$, R. Gincoul ${ }^{2}$ \\ ${ }^{1}$ Clinique Sainte-Anne Lumière, 85 Cours Albert Thomas 69003 Lyon \\ 2 Fédération des maladies de l'appareil digestif, Hôpital Ed Herriot, \\ 69008 Lyon
}

\section{Bile duct stones}

Endoscopic retrograde cholangio-pancreaticography (ERCP) has long been used as the best diagnostic method for common bile duct stones (CBDS). Moreover, it was possible to combine stone removal with endoscopic sphincterotomy (ES) during the same endoscopic session. Nevertheless it remains an invasive method, associated with complications in $5 \%$ of patients, and with a mortality rate of $0.1-0.2 \%$ [1,2] for ERCP, reaching $2.2 \%$ for ES [3 - 5]. As transabdominal ultrasound (US) or computed tomography are not particularly sensitive for the diagnosis of choledocholithiasis [6-7] an accurate diagnostic minimally invasive tool was awaited, to replace ERCP and to reserve ES for patients with CBDS.

In the past decade, helical computed tomodensitometry (hCT), endoscopic ultrasonography (EUS), and magnetic resonance cholangiopancreatography (MRCP) have improved the diagnosis of CBDS. More recently intraductal ultrasound (IDUS) has also been tested. Nevertheless ERCP is needed for this procedure and only ES should be avoided in patients without CBDS. Currently, what is the respective place of these modalities?

MRCP and hCT are non-invasive procedure. hCT is generally considered as inferior than MRCP, with a sensitivity of $85-88 \%$, specificity of $88-97 \%$, and diagnostic accuracy of $86-94 \%[8,9]$, for CBDS. Nevertheless in the first comparative study [10] the results were the same with an accuracy of $86 \%$, a sensitivity of $88 \%$ and a specificity of $75 \%$ for the two procedures. The main drawback of these two techniques is the limited spatial resolution. In the first series, stones not diagnosed by MRCP were always smaller than $10 \mathrm{~mm}$ [11 - 13]. Imaging improvements permit now the detection of calculi smaller than $5 \mathrm{~mm}$ [14] but the sensitivity remains low $(67 \%)$ for stones $<5 \mathrm{~mm}$ as for MRCP than for hCT [10]. Moreover, MRCP is contraindicated in patients with a permanent pacemaker or metallic clips, and claustrophobic patients cannot undergo the examination, when $\mathrm{hCT}$ is not applicable to patients allergic to contrast medium.

EUS offers higher resolution than MRCP or hCT ( 0.1 versus 1 $\mathrm{mm}$ ). The accuracy of EUS is excellent with sensitivity, specificity and accuracy ranging from 81 to $98 \%, 93$ to $100 \%$ and 93 to $99 \%$ respectively [15-23]. It is a minimally invasive method $[20,24]$ with a lower failure rate than ERCP ${ }^{16}$. The specificity of EUS in ruling out the presence of CBDS is very high, until $98 \%$ [25]. De-

Correspondence: Bertrand Napoleon · 35 Rue Bataille 69008 LYON · Phone: 0033478781051 - Fax: 0033478740792 · E-mail: bertrand.napoleon@libertysurf.fr 
tection of bile duct sludge as well as minilithiasis, often missed by the other imaging techniques [26], is possible with EUS. The type of echoendoscope seems of no importance. Results are as good with radial echoendoscopes as with linear scopes [21] or with extraductal catheter probe EUS [27].

The accuracy of IDUS for the diagnosis of CBDS is also very high, reaching $100 \%$ [ $28-29]$. The size of the probe $(2 \mathrm{~mm})$ allows an insertion inside the bile duct during an ERCP. The high frequency $(20 \mathrm{MHz})$ provides higher resolution than conventional EUS. Moon et al in 2005 compared the sensitivity of IDUS and MRCP to the results of ERCP + ES and stone removal [28]. For the detection of CBDS the sensitivity of IDUS and ERCP was $95 \%$ and $80 \%$ respectively. The clinical utility of IDUS was evaluated by Catanzaro et al [29]. The performance was compared with results of ERCP. IDUS found CBDS in 8 out of 21 patients with normal cholangiogram. ES confirmed the diagnosis in 7 patients. IDUS also avoided unnecessary ES as no lithiasis was found in $36 \%$ of patients with a positive finding on ERCP. The main drawback of IDUS remains the morbidity of ERCP which must be performed at the same time.

Very good comparative studies between these procedures are rare. In fact, to compare the performance of each technique, some parameters have to be considered. Spontaneous stone migration between the two examinations can lead to false-positive results. Stone migration has been found to have occurred in $21 \%$ of patients within 1 month [30]. Ideally, the 'gold standard' examination should be performed as soon as possible (at least during the following 2 days) after the evaluated technique. Second, ERCP (or peroperative cholangiography) is not the best 'gold standard' as its sensitivity to diagnose CBDS is around 90\% [18]. Two different approaches should be considered as the most sensitive and specific : - systematic ERCP, ES and instrumental exploration of the CBD (with a Dormia basket or balloon) [18]; depending of the result of the diagnostic tool : association of ERCP, ES, and bile duct exploration when a stone is evidenced or 6 months clinical follow-up when a stone has been excluded $[20,22,23,31]$. More recently the excellent results of ERCP with IDUS [10] should also lead to a third 'gold standard'.

In the first comparative studies, EUS was found to be superior to MRCP $[10,32,33]$ or hCT $[9,10,19]$ to detect CBDS. Improvements in imaging modalities may modify these findings: in the most recent series, the specificity of EUS and MRCP were found to be equal $(97 \%)$ when sensitivity was slightly greater with EUS than with MRCP (94\% versus $88 \%$, respectively) [14]. These good results need to be confirmed in largest series. At present, EUS can be considered to be more accurate than hCT or MRCP, especially for smaller stones.

A considerable reduction in the number of inappropriate ERCP is obtained with the use of alternative imaging procedures $[22,23]$. Given that the accuracy of EUS is greater than that of MRCP or $\mathrm{hCT}$, at least in patients with microlithiasis $[13,32,33]$ EUS should replace diagnostic ERCP. Whenever EUS is contraindicated (total or Billroth II gastrectomy, digestive stenosis...), MRCP or hCT might be proposed. ERCP should be used in patients already known to have CBDS (shown by US, for example) or in patients with previous ES. IDUS cannot be proposed as a first step proce- dure because of the ERCP morbidity. It might be reserved to patients in whom CBDS have been found at EUS or MRCP, but not at ERCP.

Is it necessary to perform a systematic minimally invasive diagnostic procedure before ERCP?

Patients suspected of having CBDS on clinical and laboratory criteria and/or US findings can be ranged into risk classes from low to high $[34,35]$. Less than $30 \%$ of patients classified as being at moderate risk have CBDS [20,31] whereas the proportion of high-risk patients that actually have CBDS is around 70\% $[18,19,31,36]$. For moderate- and low-risk patients, the general consensus is to perform a first-line diagnostic (after US). Thus, EUS could be the ideal alternative, selecting only those patients with bile duct stones for ERCP/ES. In high-risk patients ERCP is generally proposed as the first-line approach $[20,22,31,35,37]$. Nevertheless, as it is impossible completely to avoid unnecessary ERCP investigations on clinical or biochemical criteria, some authors support EUS as a first-line approach $[23,38,39]$. In the recent series of Liu et al [38] 140 patients presenting acute pancreatitis suspected to be of biliary origin were prospectively randomized. In the first group, EUS was followed by ERCP when needed, and in the second group, ERCP was performed immediately. EUS had a higher successful examination rate and a higher sensitivity in the detection of cholelithiasis. The morbidity was higher in the ERCP group (14\% of complications) than in the EUS group (7\%) but it was not significant. Then the best choice, also in high risk patients, should be to perform EUS followed, during the same endoscopic procedure, with ERCP and ES when a stone is evidenced.

Regard to cost-effectiveness, first line EUS vs first line ERCP has been studied in two situations $[23,40]$. In the context of laparoscopic cholecystectomy [23] EUS was performed for all patients with suspected CBDS followed by ERCP and ES when stone was evidenced. The cost of this approach was compared with the cost of a strategy proposing ERCP as first step. The mean cost for patients managed by the EUS-based strategy was significantly less than the theoretical mean cost for patients undergoing ERCP approach. In the context of acute biliary pancreatitis, Romagnuolo et al. [40] constructed a modeling standard care for non severe (selective ERCP) and severe pancreatitis (ERCP with sphincterotomy and balloon sweep). EUS was dominant in severe pancreatitis whereas it was slightly more costly in non severe (but associated with fewer ERCPs and ERCP-related complications). So, even if the estimation of cost is different depending of the countries and healthcare systems, EUS as first diagnostic tool seems to be the most effective not only for clinical impact but also for economic target. Further studies are necessary to confirm that the EUS-based strategy is also cost-effectiveness in the sub-group of high risk patients.

\section{Gallbladder stones}

US is very accurate for the diagnosis of gallbladder stones, with a sensitivity and a specificity of $97 \%$ and $95 \%$, respectively [41]. Nevertheless sensitivity is lower in some conditions: - when US is hampered by technical problems as in obese patients; - when 
stones are too small $(<2 \mathrm{~mm})$ or located in difficult area to examine (cystic duct). When there is discordance between clinical presentations (biliary-type pain) and negative US findings, the use of bile crystal analysis has long been the most common approach [42]. For ten years some series have been published confirming the interest of EUS in diagnosing small CBD stones or sludge in the gallbladder. The results were excellent compared with crystal bile analysis $[43,44]$. EUS was at least as accurate than crystal bile analysis [43] or even better [44] with a sensitivity of $96 \%$ and $67 \%$ respectively ( $p<0.03$ ) when specificity was not different ( 86 and $91 \%$, respectively). EUS has also been proved effective to diagnose small gallbladder stones in case of acute pancreatitis. So gallstones were found by EUS in 14 of 18 patients with negative findings on US by Liu et al. [25] in 2000. These results were confirmed in a larger series [26]. EUS identified unnoticed gallbladder lithiasis (sludge or very small stones) in $40 \%$ of 168 patients referred with a diagnosis of idiopathic pancreatitis. This underlines the importance of EUS for the diagnosis of gallbladder lithiasis.

\section{Acute pancreatitis}

In patient with acute pancreatitis of unknown origin after usual explorations (history, US, biochemical tests ...) EUS is mandatory $[25,26,45,46]$. It can help to diagnose biliary pancreatitis (see above), to evidence rare causes as small tumors or intraductal pancreatic mucinous tumors (IPMT) [26] or to suggest an underlying early chronic pancreatitis (CP). Overall, EUS should be able to find a cause for the 'idiopathic' acute pancreatitis in $80 \%$ of patients [26]. The delay needed between EUS and acute pancreatitis is difficult to assess: when the main question concerns a possible CBDS, EUS should be done quickly to avoid unnecessary diagnosis ERCP (see above). When the question is to find an origin not evidenced by conventional tests, inflammatory parenchyma modifications should preclude to evidence a small tumor or IPMT and to conclude to an early CP. Sludge should also be overestimated in fasting patients. The most effective is probably to perform EUS 2 to 4 weeks after the attack in recovering and normally eating patients. When modifications of the parenchyma remain, the differential diagnosis between inflammatory consequences of the acute pancreatitis or modifications due to an underlying early CP can be difficult. In some cases a new EUS performed 3 to 6 months later should be useful to conclude. There is no interest to wait for recurrent attacks of acute pancreatitis to perform EUS: Yusoff et al compared the results of EUS in patients having a single episode of acute pancreatitis versus patients with recurrent pancreatitis ${ }^{47}$. EUS findings were not more frequent for patients with recurrent pancreatitis than for the former. The most common findings was $\mathrm{CP}$ which was found twice as frequent in patients with recurrent episodes vs. a single episode of "idiopathic" pancreatitis.

\section{Chronic pancreatitis}

The main question for $\mathrm{CP}$ is to diagnose an early symptomatic disease. This is especially important in case of acute pancreatitis or chronic epigastric pain of unknown origin (no abnormalities on conventional diagnostic methods, such as US, CT, MRI, and on non invasive pancreatic function tests). In such situations diagnosis of $\mathrm{CP}$ remains a difficult challenge because of different considerations:

- no 'gold standard' test is available.

No morphologic neither functional test is a perfect diagnostic test. Some of them are more sensitive when others are more specific. When comparing EUS to another test the results must be interpreted depending of the characteristics of the 'gold standard' test chosen. The risk is to create false positive results if it is highly sensitive or false negative if it is highly specific.

- CP is a progressive disease

It should be necessary, to avoid false positive diagnosis secondary to an unsatisfactory sensitivity of the test chosen as 'gold standard' to re-evaluate the diagnosis of $\mathrm{CP}$ some years later. Such series would certainly be the most powerful. But they are rare as more difficult to conduct.

- no specific criteria

Ideally EUS should detect subtle changes appearing early during the disease and different from the possible variations of a normal pancreas. The modifications noticed in the pancreas in case of $\mathrm{CP}$ can reach the parenchyma or the ducts. Wiersema et al [48] defined some criteria which can be seen. The usual scoring system includes parenchyma changes (hyperechoic foci $>3 \mathrm{~mm}$, hyperechoic strands, and lobularity) and ductal changes (hyperechoic main pancreatic duct wall, dilatation of the main pancreatic duct, irregular contour of main pancreatic duct, visible side branches, cysts or calcifications). Nevertheless none of these criteria is entirely specific. Moreover, if some signs are probably more specific than others (calcification vs hyperechoic main pancreatic duct wall...) it has not been evaluated.

- necessity of a threshold

As no sign is specific the diagnosis is depending of an addition of positive items. Nevertheless, in this situation, the diagnostic threshold will completely modify the sensitivity and the specificity of the test. If the threshold is low, the test should over-diagnose the disease (false-positive results). If it is high, the test should under-diagnose it (false-negative results). Usually the presence of 1 or 2 signs is considered as non specific, 3 to 4 is equivocal, at least 5 signs is considered as probable.

- EUS criteria are operator-dependent

The inter- and intraobserver agreement of a diagnostic test is an important outcome especially for the diagnostic of subtle changes of an early disease. Wallace et al [49] tested the scoring system in a group of experienced echoendoscopists (more than 1100 lifetime pancreatic EUS examinations). The overall interobservor agreement of final diagnosis of $\mathrm{CP}$ was 0.45 (moderate agreement). Agreement was good (kappa $>0.50$ ) only for 2 items: duct dilatation and lobularity. Near-consensus agreement (> $90 \%$ ) was reached in $46 \%$ of individual EUS features. Even if these results are comparable to other commonly used endoscopic procedures such as bleeding ulcer stigmata, these results must incite any physician to be cautious when making a definite diagnosis of early CP. Computer quantitative analysis of endosonographic echogenicity of the parenchyma may provide more objective information for the diagnosis. It should overcome inter- 
observer variability in analyzing individual echo features supporting the diagnosis of $\mathrm{CP}$ as shown by Irisawa et al [50]. Further studies are nevertheless needed to confirm initial good results.

- population dependent

The interpretation of the results is depending of the population studied. Generally a normal pancreas presents a homogenous and slightly hyperechoic echopattern when the main pancreatic duct is thin and regular. In fact some modifications should be noticed in non pathologic pancreas, with a special correlation with age, as the pancreas may become atrophic, heterogeneous, with a pancreatic duct slightly dilated. The value of threshold figure of three or more criteria suggestive of $\mathrm{CP}$ was tested in patients without history of pancreaticobiliary diseases or alcohol consumption. One parenchymal or ductal abnormality at least was noted in $28 \%$ of the patients and $8 \%$ of the patients have three abnormalities. It increases with age especially after 60 . The most common abnormality was the hyperechoic stranding (18\%) followed by accentuation of the lobular pattern (11\%) and irregular ductal contour (7.5\%) [51]. Modifications can also be noticed in asymptomatic alcoholic consumers [52] or in patients with dyspepsia [53]. Whether these patients have some form of early asymptomatic CP or whether these changes are non pathologic remains questionable. In a series of patients with alcoholrelated liver cirrhosis [54], EUS detected features of CP in $25 \%$ of patients who did not progress over a mean period of 22 months. Further and longer follow-up studies of such patients are needed to provide final answer.

Depending of the patient the threshold to conclude to an early $\mathrm{CP}$ should be modified : more than 5 criteria should be necessary to conclude to the disease in asymptomatic aged patients when presence of 3 criteria should be sufficient to conclude in a symptomatic young patient.

- cause-dependant?

Generally EUS modifications do not orientate to specific cause but in diffuse autoimmune pancreatitis. EUS may demonstrate a pancreatic enlargement, associated with a multifocal narrowing of the main duct with irregular wall thickening. At the opposite calcifications and pseudocysts seem exceptional $[55,56]$.

Considering these pitfalls what results should we consider?

The sensitivity and specificity of EUS to diagnose CP has been assessed with morphologic gold standards (US, CT, ERCP) and/or a functional test (secretin test) [48,57-60]. Compared with ERCP, EUS has an overall sensitivity and specificity of $85 \%$ and $75 \%$, respectively [48,57-59]. Compared with pancreatic function testing (secretin test), EUS showed an overall sensitivity and specificity of $80 \%$ and $70 \%$, respectively $[48,57]$ or less in smaller series [60]. As no true gold standard test exists it cannot be excluded that true cases of "early" pancreatitis were diagnosed by EUS but ignored by ERCP or secretin test inducing false positive classification. This is confirmed by Kahl et al [61] in a follow-up study. They investigated a selected group of patients with recurrent upper abdominal pain and a known history of chronic alcohol abuse. From the 130 patients included, $48 \%$ had documented attacks of acute pancreatitis. ERCP was indicative of CP in $71 \%$ of cases. In 38 patients ERCP was considered as normal but EUS features of CP were present in 32 patients. During the follow-up (median 18 months), a final diagnosis of CP was finally done by a new ERCP in 22 of 32 patients (68.8\%). In this population EUS was confirmed to be more sensitive than ERCP to diagnose early CP.

Should EUS fine needle aspiration (EUS-FNA) be helpful for the diagnosis of early CP?

Hollerbach et al [62] obtained EUS-FNA cytology in 27 patients with varying disease severity as determined by ERCP. Morphologic abnormalities reached a sensitivity of $97 \%$ and a specificity of $60 \%$. The use of EUS-FNA slightly modifies the results with mainly an improvement of the negative predictive value from $75 \%$ to $100 \%$. Specificity was comparable (67\%). In a more recent study [63] true-cut biopsy (TCB) was used and results compared with EUS and ERCP. The agreement was poor between EUS and EUS-TCB and fair between ERCP and EUS-TCB. These results did not support the use of EUS-FNA in routine use.

At the opposite EUS-FNA should be of interest for the diagnosis of autoimmune pancreatitis. In the series of Farrell et al. [56], EUS guided FNA was suggestive of chronic inflammatory pancreatitis in 9 of 12 patients. As the risk of false positive FNA exists [64] the use of TCB instead of FNA may provide better diagnosis and seems safe in a short series [65].

\section{Finally is EUS really helpful when a CP is suspected?}

Probably yes to exclude the disease: when less than two criteria are present EUS can reliably allow avoiding more invasive procedure. Nevertheless further long term follow-up series are needed to confirm that EUS can really exclude the disease.

Certainly yes to confirm the disease even when other tests as ERCP are negative, but a lot of pitfalls exist and we have to remain cautious when giving this result:

- The characteristics of the patient must be considered: age, alcohol consumption, recent acute pancreatitis, chronic epigastric pain.

- The number of present items: more they are more the diagnosis is probable.

- The experience of the endosonographer.

Further studies are needed to consider the respective accuracy of each item considered in the EUS scoring system and to precise the follow-up of mild symptomatic patients with slight features suggesting of $\mathrm{CP}$.

\section{References}

${ }^{1}$ Davis WZ, Cotton PB, Arias A. ERCP and sphincterotomy in the context of laparoscopic cholecystectomy: academic and community practice patterns and results. Am J Gastroenterol 1997; 92: 597-601

2 Loperfido S, Angelini G, Benedetti G. Major early complications from diagnostic and therapeutic ERCP: a prospective multicenter study. Endoscopy 1999; 31: $125-130$

${ }^{3}$ Freeman ML, Nelson DB, Sherman S et al. Complications of endoscopic biliary sphincterotomy. N Engl J Med 1996; 335: 908 - 918 
${ }^{4}$ Sherman S, Ruffolo TA, Hawes RH et al. Complications of endoscopic sphincterotomy. Gastroenterology 1991; 101: 1068-1072

${ }^{5}$ Lambert ME, Betts CD, Hill J et al. Endoscopic sphincterotomy: the whole truth. Br J Surg 1991; 78: 473-476

${ }^{6}$ Dong B, Chen M. Improved sonographic visualization of choledocholithiasis. J Clin Ultrasound 1987; 15: 185 - 190

7 Stott MA, Farrands PA, Guyer PB et al. Ultrasound of the common bile duct in patients undergoing cholecystectomy. J Clin Ultrasound 1991; 19: $73-76$

${ }^{8}$ Neitlich JD, Topazian M, Smith RC et al. Detection of choledocholithiasis: comparison of unenhanced helical CT and endoscopic retrograde cholangiopancreatography. Radiology 1997; 203: 753 - 757

${ }^{9}$ Polkowski M, Palucki J, Regula J et al. Helical computed tomographic cholangiography versus endosonography for suspected bile duct stones: a prospective blinded study in non-jaundiced patients. Gut 1999; 45: $744-749$

${ }^{10}$ Kondo S, Isayama $\mathrm{H}$, Akahane $\mathrm{M}$ et al. Detection of common bile duct stones: comparison between endoscopic ultrasonography, magnetic resonance cholangiography, and helical-computed-tomographic cholangiography. Eur J Radiol 2005; 54: 271 - 275

${ }^{11}$ Chan WL, Chan AC, Lam WW et al. Choledocholithiasis: comparison of MR cholangiography and endoscopic retrograde cholangiography. Radiology 1996; 200: 85-89

12 Mendler MH, Bouillet P, Sautereau P et al. Value of MR cholangiography in the diagnosis of obstructive diseases of the biliary tree: a study of 58 cases. Am J Gastroenterol 1998; 93: 2482 - 2490

${ }^{13}$ Zidi SH, Prat F, Le Guen O et al. Use of magnetic resonance cholangiography in the diagnosis of choledocholithiasis: prospective comparison with the reference imaging method. Gut 1999; 44: 118-122

${ }^{14}$ Aubé C, Delorme B, Yzet T et al. MR cholangiopancreatography versus endoscopic sonography in suspected common bile duct lithiasis: a prospective, comparative study. AJR Am J Roentgenol 2005; 184: 55 62

${ }^{15}$ Amouyal P, Amouyal G, Levy P et al. Diagnosis of choledocholithiasis by endoscopic ultrasonography. Gastroenterology 1994; 106: $1062-$ 1067

16 Palazzo L, Girollet PP, Salmeron M et al. Value of endoscopic ultrasonography in the diagnosis of common bile duct stones: comparison with surgical exploration and ERCP. Gastrointest Endosc 1995; 42: $225-231$

${ }^{17}$ Shim CS, Joo JH, Park CW et al. Effectiveness of endoscopic ultrasonography in the diagnosis of choledocolithiasis prior to laparoscopic cholecystectomy. Endoscopy 1995; 27: $428-432$

18 Prat F, Amouyal G, Amouyal P et al. Prospective controlled study of endoscopic ultrasonography and endoscopic retrograde cholangiography in patients with suspected bile duct lithiasis. Lancet 1996; 347: $75-79$

${ }^{19}$ Sugiyama M, Atomi Y. Endoscopic ultrasonography for diagnosing choledocholithiasis: a prospective comparative study with ultrasonography and computed tomography. Gastrointest Endosc 1997; 45: $143-146$

${ }^{20}$ Canto MIF, Chak A, Stellato T et al. Endoscopic ultrasonography versus cholangiography for the diagnosis of choledocholithiasis. Gastrointest Endosc 1998; 47: 439-448

${ }^{21}$ Kohut M, Nowakowska-Dulawa E, Marek T et al. Accuracy of linear endoscopic ultrasonography in the evaluation of patients with suspected common bile duct stones. Endoscopy 2002; 34: 299-303

22 Napoléon B, Dumortier J, Keriven-Souquet O et al. Do normal findings at biliary endoscopic ultrasonography obviate the need for endoscopic retrograde cholangiography in patients with suspicion of common bile duct stone? A prospective follow-up of 238 patients. Endoscopy 2003; 35: $411-415$

${ }^{23}$ Buscarini E, Tansini P, Vallisa D et al. EUS for suspected choledocholithiasis: do benefits outweigh costs? A prospective, controlled study. Gastrointest Endosc 2003; 57: 510-518

${ }^{24}$ Lightdale CJ. Indications, contraindications and complications of endoscopic ultrasonography. Gastrointest Endosc 1996; 43: 15 - 19

${ }^{25} \mathrm{Liu}$ CL, Lo CM, Chan JK et al. EUS for detection of occult cholelithiasis in patients with idiopathic pancreatitis. Gastrointest Endosc 2000; 51: $28-32$

${ }^{26}$ Frossard JL, Sosa-Valencia L, Amouyal G et al. Usefulness of endoscopic ultrasonography in patients with 'idiopathic' acute pancreatitis. Am J Med 2000; 109: $196-200$
${ }^{27}$ Seifert H, Wehrmann T, Hilgers R et al. Catheter probe extraductal EUS reliably detects distal common bile duct abnormalities. Gastrointest Endosc 2004; 60: 61 -67

${ }^{28}$ Moon JH, ChoYD, ChaSW et al. The detection of bile duct stones in suspected biliary pancreatitis: comparison of MRCP, ERCP, and intraductal US. 2005; 100: 1051 - 1057

${ }^{29}$ Catanzarro A, Pfau P, Isenberg GA et al. Clinical utility of intraductal EUS for evaluation of choledocholithiasis. Gastrointest Endosc 2003; 57: $712-714$

${ }^{30}$ Frossard JL, Hadengue A, Amouyal G et al. Choledocholithiasis: a prospective study of spontaneous common bile duct stone migration. Gastrointest Endosc 2000; 51: 175 - 179

31 Berdah SV, Orsoni P, Berge T et al. Follow-up of selective endoscopic ultrasonography and/or endoscopic retrograde cholangiography prior to laparoscopic cholecystectomy: a prospective study of 300 patients. Endoscopy 2001; 33: 216-220

${ }^{32}$ Scheiman JM, Carlos RC, Barnett JL et al. Can endoscopic ultrasound or magnetic resonance cholangiopancreatography replace ERCP in patients with suspected biliary disease? A prospective trial and cost analysis. Am J Gastroenterol 2001; 96: 2900-2904

${ }^{33}$ De Ledinghen V, Lecesne R, Raymond JM et al. Diagnosis of choledocholithiasis: EUS or magnetic resonance cholangiography? A prospective controlled study. Gastrointest Endosc 1999; 49: 26-31

${ }^{34}$ Cotton PB, Baillie J, Pappas TN et al. Laparoscopic cholecystectomy and the biliary endoscopist (editorial). Gastrointest Endosc 1991; 37: 94 97

35 Cotton PB. Endoscopic retrograde cholangiopancreatography and laparoscopic cholecystectomy. Am J Surg 1993; 165: 474-478

${ }^{36}$ Abboud PA, Malet PF, Berlin JA et al. Predictors of bile duct stones prior to cholecystectomy: a meta-analysis. Gastrointest Endosc 1996; 44: $450-459$

37 Sahai AV, Mauldin PD, Marsi V et al. Bile duct stones and laparoscopic cholecystectomy: a decision analysis to assess the roles of intraoperative cholangiography, EUS and ERCP. Gastrointest Endosc 1999; 49: $334-343$

${ }^{38}$ Liu CL, FanST, LoCM et al. Comparison of early endoscopic ultrasonography and endoscopic retrograde cholangiopancreatography in the management of acute biliary pancreatitis: a prospective randomized study. Clin Gastroenterol Hepatol 2005; 3: 1238 - 1244

${ }^{39}$ Rocca R, De Angelis C, Castellino F et al. EUS diagnosis and simultaneous endoscopic retrograde cholangiography treatment of common bile duct stones by using an oblique-viewing echoendoscope. Gastrointest Endosc 2006; 63: 479-484

${ }^{40}$ Romagnuolo J, Currie G. Non invasive vs. selective invasive biliary imaging for acute biliary pancreatitis: an economic evaluation by using decision tree analysis. Gatrointest Endosc 2005 Jan; 61: 86 - 97

${ }^{41}$ Shea JA, Berlin JA, Escarce JJ et al. Revised estimates of diagnostic test sensitivity and specificity in suspected biliary tract disease. Arch Intern Med 1994; 154: 2573 - 2581

42 Ko C, Sekijima J, Lee S. Biliary sludge. Ann Intern Med 1999; 130: 301 311

${ }^{43}$ Dill JE, Hill S, Callis J et al. Combined endoscopic ultrasound and stimulated biliary drainage in cholecystitis and microlithiasis - diagnoses and outcomes. Endoscopy 1995; 27: 424-427

${ }^{44}$ Dahan P, Andant C, Levy P et al. Prospective evaluation of endoscopic ultrasonography and microscopic examination of duodenal bile in the diagnosis of cholecystolithiasis in 45 patients with normal conventional ultrasonography. Gut 1996; 38: $277-281$

${ }^{45}$ Chak A, Hawes RH, Cooper GS et al. Prospective assessment of the utility of EUS in the evaluation of gallstone pancreatitis. Gastrointest Endosc 1999; 49: 599-604

${ }^{46}$ Liu CL, Lo CM, Chan LK et al. Detection of choledocholithiasis by EUS in acute pancreatitis: a prospective evaluation in 100 consecutive patients. Gastrointest Endosc 2001; 54: 325-330

${ }^{47}$ Yusoff IF, RaymondG, SahaiAV. A prospective comparison of the yield of EUS in primary vs. recurrent idiopathic acute pancreatitis 2004 Nov; 60 (5): $673-678$

48 Wiersema MJ, Hawes RH, Lehman GA et al. Prospective evaluation of endoscopic ultrasonography and endoscopic retrograde cholangiopancreatography in patients with chronic abdominal pain of suspected pancreatic origin. Endoscopy 1993; 25: 555-564

${ }^{49}$ Wallace MB, Hawes RH, Durkalski V et al. The reliability of EUS for the diagnosis of chronic pancreatitis: interobserver agreement among experienced endosonographers. Gastrointest Endosc 2001; 53: 294-299 
${ }^{50}$ Irisawa A, Mishra G, Hernandez LV et al. Quantitative analysis of endosonographic parenchymal echogenicity in patients with chronic pancreatitis. J Gastroenterol Hepatol 2004; 19: 1199-205

${ }^{51}$ Rajan E, Clain JE, Levy MJ et al. Age-related changes in the pancreas identified by EUS: a prospective evaluation. 2005; 61: 401 - 406

${ }^{52}$ Bhutani MS. Endoscopic ultrasonography: changes of chronic pancreatitis in asymptomatic and symptomatic alcoholic patients. J Ultrasound Med 1999; 18: 455-462

53 Sahai AV, Mishra G, Penman ID et al. EUS to detect evidence of pancreatic disease in patients with persistent or nonspecific dyspepsia. Gastrointest Endosc 2000; 52: 153 - 159

${ }^{54}$ Hastier P, Buckley MJ, Francois E et al. A prospective study of pancreatic disease in patients with alcoholic cirrhosis: comparative diagnostic value of ERCP and EUS and long-term significance of isolated parenchymal abnormalities. Gastrointest Endosc 1999; 49: 705-709

55 Yang DH, Kim KW, Kim TK et al. Autoimmune pancreatitis: radiologic findings in 20 patients. Abdom Imaging 2006; 31 (1): 94-102

${ }^{56}$ Farrell JJ, Garber J, Sahani D et al. EUS findings in patients with autoimmune pancreatitis. Endosc 2004; 60: 927-936

57 Catalano MF, Lahoti S, Geenen JE, Hogan WJ. Prospective evaluation of endoscopic ultrasonography, endoscopic retrograde pancreatography, and secretin test in the diagnosis of chronic pancreatitis. Gastrointest Endosc 1998; 48: $11-17$

${ }^{58}$ Sahai AV, Zimmerman M, Aabakken L et al. Prospective assessment of the ability of endoscopic ultrasound to diagnose, exclude, or establish the severity of chronic pancreatitis found by endoscopic retrograde cholangiopancreatography. Gastrointest Endosc 1998; 48: $18-25$

${ }^{59}$ Buscail L, Escourrou J, Moreau J et al. Endoscopic ultrasonography in chronic pancreatitis: a comparative prospective study with conventional ultrasonography, computed tomography, and ERCP. Pancreas 1995; 10: $251-257$

${ }^{60}$ Chowdhury R, Bhutani MS, Mishra G et al. Comparative analysis of direct pancreatic function testing versus morphological assessment by endoscopic ultrasonography for the evaluation of chronic unexplained abdominal pain of presumed pancreatic origin. 2005; 31: 63-68

${ }^{61} \mathrm{Kahl}$ S, Glasbrenner B, Leodolter A et al. EUS in the diagnosis of early chronic pancreatitis: a prospective follow-up study. Gastrointest Endosc 2002; 55: 507-511

${ }^{62}$ Hollerbach S, Klamann A, Topalidis T, Schmiegel WH. Endoscopic ultrasonography (EUS) and fine-needle aspiration (FNA) cytology for diagnosis of chronic pancreatitis. Endoscopy 2001; 33: 824-831

${ }^{63}$ De Witt J, McGreevy K, LeBlanc J et al. EUS-guided Trucut biopsy of suspected nonfocal chronic pancreatitis. 2005; 62: 76-84

${ }^{64}$ Deshpande V, Mino-Kenudson M, Brugge WR et al. Endoscopic ultrasound guided fine needle aspiration biopsy of autoimmune pancreatitis: diagnostic criteria and pitfalls. 2005; 29: $1464-1471$

${ }^{65}$ Levy MJ, Reddy RP, Wiersema MJ et al. EUS-guided trucut biopsy in establishing autoimmune pancreatitis as the cause of obstructive jaundice. 2005 Mar; 61 (3): 467-472 